\title{
2D Heisenberg model from rotating membrane
}

\author{
Wen-Yu Wen* \\ Department of Physics and Center for Theoretical Sciences, \\ National Taiwan University, Taipei 106, Taiwan
}

\begin{abstract}
We study a rotating probe membrane in $S^{3}$ inside $A d S_{4} \times S^{7}$ background of M-theory. With (partial) gauge fixing, we show that in the fast limit the worldvolume of tensionless membrane reduces to either the $\mathrm{XXX}_{1 / 2}$ spin chain or the two-dimensional $S U(2)$ Heisenberg spin model. Later we introduce the anisotropy and couple it to the external magnetic field. We also establish the correspondence for higher dimensional (D)p-branes.
\end{abstract}

Keywords: M-theory, membrane dynamics, 2D Heisenberg model

*Electronic address: steve.wen@gmail.com 


\section{INTRODUCTION}

The anti-de Sitter/conformal field theory (AdS/CFT) correspondence has revealed deep relation between string theory and gauge theory, in particular the correspondence between IIB strings on $A d S_{5} \times S^{5}$ and $\mathcal{N}=4$ super Yang-Mills theory (SYM) at the 't Hooft's large N limit [1, 2, 3]. While the correspondence was mostly tested for those Bogomol'nyiPrasad-Sommerfield (BPS) states where partial supersymmetry protects it against quantum correction [4], semiclassical analysis on the near-BPS sector is also considered in the Ref.[5]; the integrability of this Berenstein-Madalcena-Nastase (BMN) limit allows for quantitative tests of correspondence beyond BPS states, where the energy of classical string solutions is compared to the anomalous dimension of SYM operators with large $R$-charge. On the other hand, intimate relation between SYM dynamics and integrable spin chain was realized in the Ref.[6], because the planar limit of the dilatation operator was identified with the Hamiltonian of integrable spin chains.

The string/spin chain correspondence was first demonstrated in the Ref.[7], where a classical rotating string on $S^{3}$ inside $S^{5}$ was identified with the semiclassical coherent state in the $S U(2)$ Heisenberg spin chain model. Later this identification was explored in the full $S U(3)$ sector [8] and $S L(2)$ sector [9]. A few examples of generalization were discussed in the past: the fast spinning string in the marginally deformed $A d S_{3} \times S^{3}\lfloor 10]$ and $\beta$-deformed $\mathcal{N}=4[11]$, each corresponds to the anisotropic XXZ spin chain[12, 13]. The Melvin's magnetic-deformed background was also studied in the Ref.[14].

Strings, however, are not the only elementary objects since we have learnt that there are more extended objects such as $\mathrm{D}$ (Dirichlet)p-branes in the string theory as well as membranes and five-branes in the M-theory. Those extended objects are soliton-like objects in the low energy description of supergravity, resisting any naive perturbative analysis applicable to strings. Nevertheless, they may serve as a good probe to explore non-perturbative aspects of string/M-theory, complementary to our perturbative knowledge based on strings. In addition to their non-perturbative nature, there is no unique way to gauge away degrees of freedom in the worldvolume of $p$-branes such as what we usually do to the worldsheet of strings. Despite of these apparent difficulties, it is still an educated guess that there might be similar correspondence between those extended objects and higher dimensional spin systems in some specific limit. In this paper, we take the first step to support this conjecture with concrete 
examples. In particular, with careful choice of lagrangian multipliers and proper gauge fixing, we are able to achieve the correspondence between rotating membrane and two-dimensional (2D) Heisenberg model and later generalize to arbitrary $p$ dimensions. The outline of this paper is as follows: In the section 2, a rotating tensionless membrane with gauge fixing is shown to give rise to the 2D Heisenberg model. The condition of integrability for this spin model is briefly mentioned. The anisotropy is introduced via the same deformation as shown in the Ref.[12] and vortices-like excitation is discussed. At the end, we show that upon a partial gauge fixing the action can also reduce to the $\mathrm{XXX}_{1 / 2}$ spin chain. In the section 3, the correspondence between $p$-branes and higher-dimensional spin models is also established. In particular, for D $p$-branes there exist some new features due to nontrivial dilaton field and two-form flux. We also show that the external magnetic field can be generated geometrically in all the $S U(2)$ spin models. In the conclusion, we summarize our results and comment on possible directions for future investigation. In the appendix we review the double scaling limit constructed in the Ref.[12] for the anisotropic Heisenberg model.

\section{2D HEISENBERG MODEL/MEMBRANE CORRESPONDENCE}

\section{A. $p$-brane action}

The Probe branes approach is widely used in the string/M-theory to study different aspects of brane itself or specific background, from classical to quantum levels. It takes the assumption that the back-reaction of the brane on the background is negligible. In this paper we only study a single brane in the tensionless limit, thus qualifying the probing assumption. The brane action in general falls into two types: the Dirac-Nambu-Goto type action and the Polyakov type action. The former one is intuitively simple but carries nonlinearity due to the square root. The latter one introduces non-dynamical Lagrangian multipliers in order to linearize the former action. It is, however, easier for the purpose of calculation and suitable for taking the tensionless limit. To be specific, we adopt a Polyakov type action proposed for general $p$-branes [15]:

$$
S_{p}=\int d^{2} \xi\left\{\frac{1}{4 \lambda^{0}}\left[G_{00}-2 \lambda^{j} G_{0 j}+\lambda^{i} \lambda^{j} G_{i j}-\left(2 \lambda^{0} T_{p}\right)^{2}\left|G_{i j}\right|\right]+T_{p} b_{\mu_{0} \cdots \mu_{p}} \partial_{0} X^{\mu_{0}} \cdots \partial_{p} X^{\mu_{p}},\right\}
$$

where $\vec{\xi}=\left\{\xi^{0}, \ldots, \xi^{p}\right\}$ are worldvolume coordinates and $\vec{X}=\left\{X^{0}, \cdots, X^{D}\right\}$ are $(D+1)$ dimensional target spacetime. $\lambda$ 's are the Lagrangian multipliers. $i, j=\{1, \ldots, p\}$ only run 
for spatial indices. $G_{i j} \equiv \partial_{i} X^{\mu} \partial_{j} X^{\nu} g_{\mu \nu}$ is the pull back metric on the worldvolume and $|G|$ is for determinant. This action can be shown equivalent to the Dirac-Nambu-Goto type action with the following equations of motion of $\lambda$ 's substituted in,

$$
\begin{aligned}
& G_{00}-2 \lambda^{j} G_{0 j}+\lambda^{i} \lambda^{j} G_{i j}+\left(2 \lambda^{0} T_{p}\right)^{2}\left|G_{i j}\right|=0, \\
& G_{0 j}-\lambda^{i} G_{i j}=0 .
\end{aligned}
$$

Without loss of generality, we may set $\lambda^{i}=0$ but $\lambda^{0} \neq 0$. Then the action is simplified and equations of motion of $\lambda$ 's reduce to the Virasora-like constraints

$$
\begin{aligned}
& G_{00}+\left(2 \lambda^{0} T_{p}\right)^{2}\left|G_{i j}\right|=0, \\
& G_{0 j}=0
\end{aligned}
$$

\section{B. Gauge fixing and tensionless limit}

We will restrict our discussion on membranes in the M-theory for the moment and come back to general p-branes in the next section. We first fix the gauge as follows,

$$
X^{0}=\kappa \xi^{0}, \quad X^{9}=c_{2} \xi^{2}, \quad X^{10}=c_{1} \xi^{1}
$$

and the determinant decomposes into

$$
\left|G_{i j}\right|=\left|\tilde{G}_{i j}\right|+c_{2}^{2} g_{99} \tilde{G}_{11}+c_{1}^{2} g_{[10][10]} \tilde{G}_{22}+c_{1}^{2} c_{2}^{2} g_{99} g_{[10][10]},
$$

where the $\tilde{G}_{i j}$ is the pull back metric of subspace $\left\{X^{1}, \cdots, X^{8}\right\}$. Then the action becomes

$$
\begin{aligned}
& S_{m_{2}}=\int d^{3} \xi\left\{\frac{1}{4 \lambda^{0}}\left[G_{00}-\left(2 \lambda^{0} T_{2}\right)^{2}\left|\tilde{G}_{i j}\right|-\mathrm{g}_{1}^{2} \tilde{G}_{11}-\mathrm{g}_{2}^{2} \tilde{G}_{22}-\frac{\mathrm{g}_{1}{ }^{2} \mathrm{~g}_{2}{ }^{2}}{2 \lambda^{0} T_{2}}\right]+T_{2} b_{\mu \nu \lambda} \partial_{0} X^{\mu} \partial_{1} X^{\nu} \partial_{2} X^{\lambda}\right\}, \\
& \mathrm{g}_{1} \equiv 2 \lambda^{0} c_{2} T_{2} \sqrt{g_{99}}, \quad \mathrm{~g}_{2} \equiv 2 \lambda^{0} c_{1} T_{2} \sqrt{g_{[10][10]}} .
\end{aligned}
$$

In particular, we are looking for the limit where the membrane is tensionless but effective couplings $\mathrm{g}_{\mathrm{i}}$ 's are finite. This can be achieved by sending $T_{2} \rightarrow 0$ and $c_{i} \rightarrow \infty$ but keeping their product finite. With this gauge choice and scaling, we only pay attention to the local property of the membrane regardless its global topology. In the next section, we will see that the tensionless membrane in this gauge fixing provides an appropriate setting for the 2D Heisenberg Model. 


\section{2D Heisenberg model from fast membrane}

In order to reproduce the Heisenberg model, a fast membrane limit has to be taken in analogy to the Ref.[7]. We first make the membrane rotate in one angular direction then take the fast limit. In this limit, we send $\kappa \rightarrow \infty$ and $\dot{X} \rightarrow 0$ but keep $\kappa \dot{X}$ finite, here $\dot{X}$ is partial derivative w.r.t. $\xi^{0}$. The physics behind implies that at this time scale, along those direction transverse to the rotation plane, the membrane moves very slowly and can be seen as almost frozen. Although the conjugated energy and momentum at this scale in fact blow up, the finite part of worldvolume action is recognized as the sigma model of Heisenberg spin system. To illustrate this, we will assume that a tensionless probe membrane, constructed in the previous subsection, rotates inside a $S^{3}$, which could be part of the eleven dimensional vacuum of M-theory, such as $A d S_{4} \times S^{7}$. The metric $g_{99}, g_{[10][10]}$ are assumed to take constant values on the sphere for simplicity throughout the paper, but this assumption can be easily relaxed. The relevant background metric after rotating, i.e. $\alpha \rightarrow t+\alpha$, is given by

$$
d s^{2}=\frac{1}{4}\left[2 d t d \alpha+d \alpha^{2}+d \beta^{2}+d \gamma^{2}+2 \cos \beta d \alpha d \gamma+2 \cos \beta d t d \gamma\right] .
$$

After taking the fast limit, the finite worldvolume action with pull back metric reads ${ }^{1}$

$$
S_{m_{2}}=\frac{1}{16 \lambda^{0}} \int d^{3} \xi\left\{2 \kappa \dot{\alpha}+2 \kappa \cos \beta \dot{\gamma}-\sum_{i=1}^{2} \mathrm{~g}_{i}{ }^{2}\left[\left(\partial_{i} \alpha\right)^{2}+\left(\partial_{i} \beta\right)^{2}+\left(\partial_{i} \gamma\right)^{2}+2 \cos \beta \partial_{i} \alpha \partial_{i} \gamma\right]\right\}
$$

Notice that only derivatives of first order in $\xi^{0}$ and second order in $\xi^{i}$ are survived in this limit, which is the very differential strucutre underlying the Heisenberg spin model. Applying the constraint (4), one achieves the action of Heisenberg model:

$$
S_{m_{2}}=\frac{1}{16 \lambda^{0}} \int d^{3} \xi\left\{2 \partial_{t} \alpha+2 \cos \beta \partial_{t} \gamma-\sum_{i=1}^{2} \mathrm{~g}_{i}^{2}\left[\left(\partial_{i} \beta\right)^{2}+\sin ^{2} \beta\left(\partial_{i} \gamma\right)^{2}\right]\right\},
$$

or the Hamiltonian after the Legendre transformation,

$$
H_{2 D}=\frac{\mathrm{g}^{2}}{16 \lambda^{0}} \int d \xi^{1} d \xi^{2}(\partial \vec{S})^{2}
$$

Here we have made $\mathrm{g}_{\mathrm{i}}=\mathrm{g}$ for the universal coupling and defined a $S O(3)$ vector $\vec{S}=$ $(\sin \beta \sin \gamma, \sin \beta \cos \gamma, \cos \beta)$, which smoothly rotates as we move around the $\xi^{1}-\xi^{2}$ plane.

\footnotetext{
${ }^{1}$ The complete action also includes a divergent constant term which is inversely proportional to $T_{2}$. It is generically finite but seen as an artifact in the tensionless limit. Anyway, this constant term will not affect the equations of motion.
} 
Strictly speaking, this Hamiltonian is the long wavelength or continuous limit of that of the discrete lattice model, where the only interaction is among nearest neighbors. Therefore this is straightforward generalization of one-dimensional spin chain to higher dimensions. The nearest diagonal sites are next-nearest neighbors and interaction among them only appears in the second loop mixing. In another words, that mixing enters the nonlinear sigma model of worldsheet/worldvolume only via higher order correction, if desired.

\section{Membrane excitation and integrability}

In the Ref.[16], magnon-like excitation was studied for the membrane in the same Polyakov action. In our limit, the membrane action is greatly simplified and excitation on the membrane has its description in the corresponding Heisenberg model. The equation of motion derived from the Eq.(A2) is the very $(1+2)$-dimensional Landau-Lifshitz equation, i.e.

$$
\partial_{t} \vec{S}=\mathrm{g}^{2} \vec{S} \times \partial^{2} \vec{S}
$$

which, different from its $(1+1)$-dimensional counterpart, is not integrable in general. This system is gauge equivalent to the $(1+2)$-dimensional non-linear Schrödinger-type equation[17]. It has been shown that, however, for the following travelling wave ansatz it is still integrable,

$$
\vec{S}\left(\vec{\xi}, \xi^{0}\right)=\vec{S}\left(\vec{\xi}-\vec{v} \xi^{0}\right), \quad \vec{\xi}=\left(\xi^{1}, \xi^{2}\right) .
$$

We will follow the Ref.[18] to discuss its integrability by taking the advantage of isomorphism $S O(3) \simeq S U(2)$. To do so, we set $\mathrm{g}=1$ for simplicity and rewrite $S=S^{a} \hat{\sigma}^{a}$, where $\hat{\sigma}^{a}$ are the Pauli matrices and introduce complex variables $z=\frac{1}{2}\left(\xi^{1}+i \xi^{2}\right)$ and $\eta=v^{1}+i v^{2}$, and their complex conjugates $\bar{z}, \bar{\eta}$. Then one can form a Lax pair $\left(C^{-}, C^{+}\right)$with a $2 \times 2$ complex matrix $\Psi$,

$$
\begin{aligned}
\partial_{z} \Psi & =-C^{-} \Psi \\
\partial_{\bar{z}} \Psi & =C^{+} \Psi \\
C^{-} & \equiv \frac{\left[S, \partial_{z} S\right]}{2 i(x-i)}+\frac{x \bar{v} S}{(x-i)^{2}}, \quad C^{+} \equiv \frac{\left[S, \partial_{\bar{z}} S\right]}{2 i(x+i)}+\frac{x v S}{(x+i)^{2}} .
\end{aligned}
$$

The integrability condition thus reads $C_{z}^{+}+C_{\bar{z}}^{-}-\left[C^{+}, C^{-}\right]=0$. The arbitrary real spectral parameter $x$ relates to a rotation on $v$ in the following way,

$$
v^{\prime}=e^{-i \theta} v, \quad x= \pm \cot \theta / 2 .
$$


In fact, one can construct a new travelling wave solution with velocity $\overrightarrow{v^{\prime}}$

$$
\overrightarrow{S^{\prime}}=\overrightarrow{S^{\prime}}\left(\vec{\xi}-\overrightarrow{v^{\prime}} \xi^{0}\right)
$$

where $S^{\prime}=\Psi^{\dagger} S \Psi$ with $S^{\prime}=S^{a} \hat{\sigma}^{a}$.

\section{E. Anisotropy and vortex dynamics}

It has been shown in the Ref.[12] that in the limit of fast string and small deformation, a one-parameter deformation on the target $S^{3}$ reproduces the anisotropic Heisenberg spin chain $X X Z_{1 / 2}$. This generalization is also applicable to the fast membrane considered in the present paper. We will summarize the derivation in the Appendix and only present the result here. The modified Landau-Lifshitz equation is now with an anisotropic $3 \times 3$ matrix $\mathcal{J}$, i.e.

$$
\begin{aligned}
& \partial_{t} \vec{S}=\mathrm{g}^{2} \vec{S} \times \partial^{2} \vec{S}+\vec{S} \times \mathcal{J} \vec{S}, \\
& \mathcal{J}_{11}=\mathcal{J}_{22}=1, \quad \mathcal{J}_{33}=1-\delta .
\end{aligned}
$$

$\delta$ is the deformed parameter which rescales the $U(1)$ fiber in the Hopf fibration of $S^{3}$. It reduces to $X Y$-model and isotropic $X X X_{1 / 2}$ model for $\delta=1$ and $\delta=0$, respectively. The same model has been applied to the study of two-dimensional magnets for decades and we expect it could be carried over, with some caution, to the study of tensionless membrane in the fast/small deformation limit. The excitation in the two-dimensional system is much richer than that in the one-dimensional spin chain. To our particular interests, topological objects such as vortices can be excited[19, 20]. The vortices here carry two topological charges under the homotopy group $\pi_{1}$ and $\pi_{2}$, respectively. They are the vorticity, $q= \pm 1, \pm 2, \cdots$ and the polarization $p$, where $p= \pm 1$ for non-planar excitation and $p=0$ for planar one. The dynamics of vortices is governed by both the Coulomb force and Magnus force. The latter is a gyro force coupled to the vortex velocity, thus its behavior is nonNewtonian. A single static vortex is non-local for its naked topological charge. A vortexantivortex pair, however, can be localized in the form of dipole charge. Other than vortices, one also expects the meson-like excitation, magnons, appears as usual. A domain wall could even form if several of them are excited coherently.

In fact, the shape of vortex can be given by a more generic travelling wave ansatz[20],

$$
\vec{S}\left(\vec{\xi}, \xi^{0}\right)=\vec{S}\left(\vec{\xi}-\vec{x}, \dot{\vec{x}}, \ddot{\vec{x}}, \cdots, \vec{x}^{(n)}\right),
$$


where $\vec{x}$ is the trajectory of vortex center. Given the anisotropic Hamiltonian $H=H_{2 D}+$ $\frac{\mathrm{g}^{2}}{16 \lambda^{0}} \int d^{2} \xi \delta\left(S^{3}\right)^{2}$, the Eq.(17) can be rewritten as

$$
\frac{\partial \vec{S}}{\partial \xi^{0}}=-\vec{S} \times \frac{\partial H}{\partial \vec{S}},
$$

which yields an $(n+1)$-th order differential equation for $\vec{x}\left(\xi^{0}\right)$. We direct the readers to the Ref.[20] for more discussion on dynamics of those excitations.

\section{F. Spin chain from partial gauge fixing}

In the previous discussion we have succeeded in establishing the correspondence between the 2D Heisenberg model and a rotating membrane, here we would like to explore another possible correspondence associated with the membrane. In the case of partial gauge fixing, one may instead obtain the $\mathrm{XXX}_{1 / 2}$ spin chain from the same limit. To illustrate this, let us consider the following partial gauge fixing,

$$
X^{0}=\kappa \xi^{0}, \quad X^{10}=c \xi^{1},
$$

and the determinant decomposes into

$$
\left|G_{i j}\right|=\left|\tilde{G}_{i j}\right|+c^{2} g_{[10][10]} \tilde{G}_{22},
$$

where the $\tilde{G}_{i j}$ is now the pull back metric of subspace $\left\{X^{1}, \cdots, X^{9}\right\}$. Then the action becomes

$$
\begin{aligned}
& S_{m_{2}}=\int d^{3} \xi\left\{\frac{1}{4 \lambda^{0}}\left[\tilde{G}_{00}-\left(2 \lambda^{0} T_{2}\right)^{2}\left|\tilde{G}_{i j}\right|-\mathrm{g}^{2} \tilde{G}_{22}\right]+T_{2} b_{\mu \nu \lambda} \partial_{0} X^{\mu} \partial_{1} X^{\nu} \partial_{2} X^{\lambda}\right\}, \\
& \mathrm{g} \equiv 2 \lambda^{0} c T_{2} \sqrt{g_{[10][10]}} .
\end{aligned}
$$

In the tensionless limit, i.e. $T_{2} \rightarrow 0$ but $\mathrm{g}$ kept finite, one maps the membrane to the target space $S^{3}$ and takes the fast spinning limit as before. It is not difficult to identify the resulting action with the Heisenberg $\mathrm{XXX}_{1 / 2}$ spin chain and the Hamilton is given by

$$
H_{1 D}=\frac{\mathrm{g}^{2}}{16 \lambda^{0}} \int d \xi^{2} \partial_{2} \vec{S} \cdot \partial_{2} \vec{S} .
$$

Here we see that the membrane loses its dependance on $\xi^{1}$ and behaves just like a string extending along $\xi^{2}$. From the viewpoint of double dimensional reduction where M-theory reduces to type IIA string and membranes to strings, we might think that the same spin 
chain system can also be obtained from rotating D1 string inside $S^{3}$, in comparison with the case of fundamental string[7]. However, this is puzzling since there is no D1 brane in the IIA string. A better interpretation would be that a compactified D2 brane behaves like a one-dimensional string. We are also tempted to make a connection with the Matrix model interpretation of M-theory[21], where the dynamics is translated into $(1+0)$ Matrix quantum mechanics and degrees of freedom are those of D0 particles. Here we conjecture that those D0 particles form a chain and each of them possesses a spin on $S^{2}$.

\section{HEISENBERG MODEL/ $p$-BRANE CORRESPONDENCE}

\section{A. $p$-dimensional $S U(2)$ spin model from rotating $p$-brane}

In principle, one may start with a rotating $p$-brane inside a $S^{3}$ out of $D$-dimensional spacetime. With gauge fixing as follows ${ }^{2}$ :

$$
X^{0}=\kappa \xi^{0}, \quad X^{D-p}=c_{p} \xi^{p}, \cdots, X^{D-1}=c_{1} \xi^{1}
$$

It is not difficult to show that in the tensionless limit and fast brane limit as before, we reproduce the $p$-dimensional $S U(2)$ Heisenberg model with the action,

$$
\begin{aligned}
& S_{p}=\frac{1}{16 \lambda^{0}} \int d^{p+1} \xi\left\{2 \partial_{t} \alpha+2 \cos \beta \partial_{t} \gamma-\sum_{i=1}^{p} \mathrm{~g}_{i}{ }^{2}\left[\left(\partial_{i} \beta\right)^{2}+\sin ^{2} \beta\left(\partial_{i} \gamma\right)^{2}\right]\right\}, \\
& \mathrm{g}_{i}=2 \lambda^{0} T_{p} \prod_{j=1}^{p}\left(c_{j} \sqrt{g_{[D-j][D-j]}}\right) /\left(c_{i} \sqrt{g_{[D-i][D-i]}}\right),
\end{aligned}
$$

where the limit has been taken with $T_{p} \rightarrow 0, c_{i} \rightarrow \infty$ but finite $\mathrm{g}_{\mathrm{i}}$. As for $\mathrm{D} p$ branes, more care is needed for its non-vanishing dilaton $\Phi$ and antisymmetric B-field $B_{m n}$ from the closed string sector ${ }^{3}$ as well as a $U(1)$ gauge field $F_{m n}$ from the open string sector. A Polyakov-type action is given by[15]

$$
\begin{aligned}
S_{D p}= & \int d^{p+1} \xi \frac{e^{-a \Phi}}{4 \lambda^{0}}\left[G_{00}-2 \lambda^{i} G_{0 i}+\left(\lambda^{i} \lambda^{j}-\kappa^{i} \kappa^{j}\right) G_{i j}-\left(2 \lambda^{0} T_{D p}\right)^{2}\left|G_{i j}\right|+2 \kappa^{i}\left(\mathcal{F}_{0 i}-\lambda^{j} \mathcal{F}_{j i}\right)\right] \\
& +T_{D p} c_{\mu_{0} \cdots \mu_{p}} \partial_{0} X^{\mu_{0}} \cdots \partial_{p} X^{\mu_{p}},
\end{aligned}
$$

\footnotetext{
${ }^{2}$ We always make the assumption that there is enough space for a $S^{3}$ after gauge fixing.

3 Technically speaking, dilaton field and antisymmetric B-field are in the same NS-NS sector as graviton, namely both left- and right-moving modes satisfy Neveu-Schwarz periodic condition.
} 
where $\mathcal{F}_{m n} \equiv B_{m n}+2 \pi \alpha^{\prime} F_{m n}=\partial_{[m} \mathcal{A}_{n]}$. Equations of motion for those Lagrange multipliers $\lambda^{i}, \kappa^{i}$ give the constraints,

$$
\begin{aligned}
& G_{00}-2 \lambda^{j} G_{0 j}+\left(\lambda^{i} \lambda^{j}-\kappa^{i} \kappa^{j}\right) G_{i j}+\left(2 \lambda^{0} T_{D p}\right)^{2}\left|G_{i j}\right|+2 \kappa^{i}\left(\mathcal{F}_{0 i}-\lambda^{j} \mathcal{F}_{j i}\right)=0, \\
& G_{o j}-\lambda^{i} G_{i j}=\kappa^{i} \mathcal{F}_{i j}, \\
& \mathcal{F}_{0 j}-\lambda^{i} \mathcal{F}_{i j}=\kappa^{i} G_{i j} .
\end{aligned}
$$

The choice of vanishing $\lambda^{i}, \kappa^{i}$ decouples the $\mathcal{F}$ from the metric and gives us the same Heisenberg model as in the Eq.(25). Another nontrivial choice is to keep nonzero $\kappa^{i}$. In the following, for simplicity we will discuss the case with only $\kappa^{1} \neq 0$ and $\mathcal{A}_{0}=0$. The action then simplifies as

$$
S_{D p}=\int d^{p+1} \xi \frac{e^{-a \Phi}}{4 \lambda^{0}}\left[G_{00}+\kappa^{1} \kappa^{1} G_{11}-\left(2 \lambda^{0} T_{D p}\right)^{2}\left|G_{i j}\right|\right]+T_{D p} c_{\mu_{0} \cdots \mu_{p}} \partial_{0} X^{\mu_{0}} \cdots \partial_{p} X^{\mu_{p}} .
$$

With the gauge fixing (24) and target space given in the Eq.(8), the action becomes

$S_{D p}=\int d^{p+1} \xi \frac{e^{-a \Phi}}{16 \lambda^{0}}\left\{2 \kappa \partial_{0} \alpha+2 \cos \beta \kappa \partial_{0} \gamma+\kappa^{1} \partial_{0} \mathcal{A}_{1}-\sum_{i=2}^{p} \mathrm{~g}_{i}^{2}\left(\frac{\kappa^{1}}{2 \kappa} \mathcal{F}_{1 i}\right)^{2}-\sum_{i=1}^{p} \mathrm{~g}_{i}{ }^{2}\left[\left(\partial_{i} \beta\right)^{2}+\sin ^{2} \beta\left(\partial_{i} \gamma\right)^{2}\right]\right\}$,

where those constraints in (27) have been used to replace $\alpha$ and $G_{11}$. We should take $\kappa^{1} \sim \kappa$ in order for $\mathcal{F}$ to survive the fast limit as $\kappa \rightarrow \infty$. After the Legendre transformation, one obtains the Hamiltonian of Heisenberg model with an extra magnetic field $\mathcal{F}_{1 i}$, that is,

$$
H_{D p}=\frac{1}{16 \lambda^{0}} \mathrm{~g}^{2} \int d^{p} \xi e^{-a \Phi}\left[(\partial \vec{S})^{2}+\frac{1}{2} \mathcal{F}_{1 i} \mathcal{F}^{1 i}\right] .
$$

Here introduction of the nontrivial dilaton field and two-form flux complicates the original Landau-Lifshitz equation. We simply comment that the effective coupling now varies with location and an external magnetic field goes through the $p$-volume. Their functions are to be determined by the modified equations of motion derived from the action (29).

\section{B. Lower dimensional Heisenberg model from partial gauge fixing}

In the previous section, we have learnt that with partial gauge fixing, a rotating membrane can also have its correspondence in the spin chain. The same technique can easily apply to p-branes, where a lower dimensional Heisenberg model is obtained. Here we simply mention the scheme without detail. First we partially fix the gauge,

$$
X^{0}=\kappa \xi^{0}, \quad X^{D-q}=c_{q} \xi^{q}, \cdots, X^{D-1}=c_{1} \xi^{1}
$$


where $0<q<p$. Then the determinant decomposes into

$$
\left|G_{i j}\right|=\left|\tilde{G}_{i j}\right|+\prod_{j=1}^{q}\left(c_{j}^{2} g_{[D-j][D-j]}\right) /\left(c_{i}^{2} g_{[D-i][D-i]}\right)\left|\tilde{G}_{j j}\right|+\cdots,
$$

where the $\tilde{G}_{i j}$ is the pull back metric of subspace $\left\{X^{1}, \cdots, X^{D-q-1}\right\}$. The rest terms on the right hand side is irrelevant after both tensionless and fast limit are taken. Then it is straightforward to show that $q$-dimensional Heisenberg model can be derived.

\section{Anisotropy and external magnetic field}

As shown in the Ref.[12] and summarized in the Appendix, similarly one can also deform the target $S^{3}$ for the $p$-brane. This corresponds to the $p$-dimensional anisotropic $S U(2)$ Heisenberg model. In addition to anisotropy, one may wonder if a Zeeman term can be added for interaction with the enternal field. We have just demonstrated that a local coupling and magnetic flux occurs typically for the $\mathrm{D} p$-branes. However, this magnetic flux is somehow expected from the full spectrum of string theory and takes arbitrary form unless specified by equations of motion. In fact, there is an alternative way to switch on external magnetic field, say along $z$-direction, for both string and general $p$-branes. This is done geometrically by mixing two angles of $S^{3}$ unevenly. Start with the usual parametrization,

$$
d s^{2}=d \theta^{2}+\sin ^{2} \theta d \psi_{1}^{2}+\cos ^{2} \theta d \psi_{2}^{2}
$$

and then perturb the mixing matrix with a deformation parameter $b$, such that

$$
\left(\begin{array}{c}
\theta \\
\psi_{1} \\
\psi_{2}
\end{array}\right)=\left(\begin{array}{ccc}
1 / 2 & 0 & 0 \\
0 & 1 / 2(1+b) & -1 / 2 \\
0 & 1 / 2(1-b) & 1 / 2
\end{array}\right)\left(\begin{array}{l}
\beta \\
\alpha \\
\gamma
\end{array}\right) .
$$

The metric after transformation becomes

$$
d s^{2}=\frac{1}{4}\left[d \beta^{2}+d \alpha^{2}+d \gamma^{2}+2 \cos \beta d \alpha d \gamma-2 b \cos \beta d \alpha^{2}-2 b d \alpha d \gamma+b^{2} d \alpha^{2}\right] .
$$

After taking the fast limit, in the same spirit of the small deformation limit, we then send $b \rightarrow 0$ but only keep $\kappa^{2} b$ finite. This results in an additional term $-2 b \cos \beta$ to the action (25), here $\kappa$ has been absorbed into redefinition of $\kappa \dot{X} \rightarrow \dot{X}$ and $\kappa^{2} b \rightarrow b$. It can be further

put into the form $-\vec{B} \cdot \vec{S}$ and precisely interpreted as the interaction with external magnetic 
field $\vec{B}=2 b \hat{z}$. This uneven mixing of angles was already observed in [22], there appears as mixing of angular velocities. In fact, it can be shown that our double scaling limit of small deformation is equivalently to their decoupling limit.

\section{CONCLUSION}

We have realized in the fast membrane limit the 2D Heisenberg model, which is integrable at least for the ansatz of travelling wave. In the Ref.[23], the author found several types of membrane embedding into the $A d S_{4} \times S^{7}$ background, which are related to the Neumann and Neumann-Rosochatius integrable systems. Our result supports his conjecture that there might have various kinds of integrable system dual to membranes in the M-theory.

In this paper, we also proposed the correspondence between $p$-branes and $p$-dimensional Heisenberg spin model. For D p-branes, in particular, the nontrivial dialton field generates a position-varying coupling and the two-form flux couples to the system magnetically. Later we also provide a geometric realization of external magnetic field in the $S U(2)$ Heisenberg model in arbitrary dimension.

Several directions may deserve further investigation. Here we only mention some of them: In the string/spin chain correspondence, one is able to identify the anomalous dimension of single trace operator $\operatorname{Tr}(Z Z \ldots Z)$, calculated from the dual super Yang-Mills theory, with the Hamiltonian of spin chain. The two or higher dimensional spin model may not have such a correspondence due to lacking the knowledge of hypersurface operators. However, the spin chain model obtained from partial gauge fixing can still have its correspondence on the dual field theory side. In the present case with membranes, calculation of single trace operators in a three-dimensional $\mathcal{N}=8$ super Yang-Mills $\left(\mathrm{CFT}_{3}\right.$ as its IR fixed point) may support this correspondence.

In the view of $2 \mathrm{D}$ lattice, it is tempted to also switch on interaction among diagonal sites, which counts as next-nearest neighbors as previously mentioned. It could be interesting to investigate the way it appears in the worldvolume action, in comparison to the $\alpha^{\prime}$ correction in the string worldsheet.

Finally, in the Ref.[24], we have learnt that under a partial gauge fixing, a membrane can be seen as a perturbation around string like configuration, where the membrane tension acts like the coupling constant. It is tempting to relax our tensionless limit and study the 
deviation from the Heisenberg model perturbatively.

\section{ACKNOWLEDGEMENTS}

I am grateful to invitation of NCTS/NTHU to present this work at its final stage. I would

like to thank K. Furuuchi, P. M. Ho, S. Teraguchi, D. Tomino, Q. S. Yan, and S. Zeze for useful discussion and comments. The author is supported in part by the Taiwan's National Science Council under Grant No. NSC95-2811-M-002-013.

\section{APPENDIX A: ANISOTROPIC HEISENBERG MODEL FROM DEFORMED $S^{3}$}

The deformed metric is given by

$$
d s^{2}=\frac{1}{4}\left[-d t^{2}+d \beta^{2}+\sin ^{2} \beta d \alpha^{2}+(1-\delta)(d \gamma+\cos \beta d \alpha)^{2}\right]
$$

We request $0 \leq \delta \leq 1$ to avoid non-unitary gauge field $(\delta<0)$ and closed time-like geodesics $(\delta>1)$. For trivial $\delta=0$, one recovers the unit round $S^{3}$ as Hopf fibration. For maximal $\delta=1$, the $S^{1}$ fiber degenerates and we are left with $S^{2}$.

After sending $\alpha \rightarrow \alpha+t$ and taking fast limit as the undeformed one, then the relevant part becomes

$$
\begin{aligned}
& S_{m_{2}}^{\prime}= \frac{1}{16 \lambda^{0}} \int d^{3} \xi\left\{-\delta \cos ^{2} \beta \kappa^{2}+2 \kappa \dot{\alpha}+2 \kappa \cos \beta \dot{\gamma}-\sum_{i=1}^{2} \mathrm{~g}_{i}{ }^{2}\left[\left(\partial_{i} \beta\right)^{2}+\Delta_{\delta}\left(\partial_{i} \gamma\right)^{2}\right]\right\}, \\
& \Delta_{\delta} \equiv \frac{(1-\delta) \sin ^{2} \beta}{1-\delta \cos ^{2} \beta} .
\end{aligned}
$$

In order to keep only the anisotropic term, we have to take the small deformation limit by sending $\delta \rightarrow 0$ but keep $\kappa^{2} \delta$ finite. In this limit, the $\Delta_{\delta} \rightarrow \sin ^{2} \beta$ as desired. After both fast and small deformation limits are taken, it is convenient to rescale $\kappa^{2} \delta \rightarrow \delta$ and $\kappa \dot{X} \rightarrow \dot{X}$. The equations of motion for $\beta$ and $\gamma$ are then given by

$$
\begin{aligned}
& \mathrm{g}^{2} \beta^{\prime \prime}-\sin \beta \dot{\gamma}-\mathrm{g}^{2} \sin \beta \cos \beta\left(\gamma^{\prime}\right)^{2}+\delta \sin \beta \cos \beta=0 \\
& \sin \beta \dot{\beta}+\mathrm{g}^{2}\left(\sin ^{2} \beta \gamma^{\prime}\right)^{\prime}=0,
\end{aligned}
$$

which gives rise to the general Landau-Lifshitz equation,

$$
\begin{aligned}
& \partial_{t} \vec{S}=\mathrm{g}^{2} \vec{S} \times \partial^{2} \vec{S}+\vec{S} \times \mathcal{J} \vec{S}, \\
& \mathcal{J}_{11}=\mathcal{J}_{22}=1, \quad \mathcal{J}_{33}=1-\delta,
\end{aligned}
$$


with the same spin vector $\vec{S}=(\sin \beta \cos \gamma, \sin \beta \sin \gamma, \cos \beta)$.

This general Landau-Lifshitz equation can be seen as continuous limit of the inhomogeneous Heisenberg spin chain model.

[1] J. M. Maldacena, "The large N limit of superconformal field theories and supergravity," Adv. Theor. Math. Phys. 2, 231 (1998) [Int. J. Theor. Phys. 38, 1113 (1999)] arXiv:hep-th/9711200.

[2] S. S. Gubser, I. R. Klebanov and A. M. Polyakov, "Gauge theory correlators from non-critical string theory," Phys. Lett. B 428, 105 (1998) arXiv:hep-th/9802109].

[3] E. Witten, "Anti-de Sitter space and holography," Adv. Theor. Math. Phys. 2, 253 (1998) arXiv:hep-th/9802150].

[4] O. Aharony, S. S. Gubser, J. M. Maldacena, H. Ooguri and Y. Oz, "Large N field theories, string theory and gravity," Phys. Rept. 323, 183 (2000) arXiv:hep-th/9905111.

[5] D. Berenstein, J. M. Maldacena and H. Nastase, "Strings in flat space and pp waves from N = 4 super Yang Mills," JHEP 0204, 013 (2002) arXiv:hep-th/0202021].

[6] N. Beisert, S. Frolov, M. Staudacher and A. A. Tseytlin, "Precision spectroscopy of AdS/CFT," JHEP 0310, 037 (2003) [arXiv:hep-th/0308117].

[7] M. Kruczenski, "Spin chains and string theory," Phys. Rev. Lett. 93, 161602 (2004) arXiv:hep-th/0311203.

[8] R. Hernandez and E. Lopez, "The SU(3) spin chain sigma model and string theory," JHEP 0404, 052 (2004) arXiv:hep-th/0403139.

[9] S. Bellucci, P. Y. Casteill, J. F. Morales and C. Sochichiu, "SL(2) spin chain and spinning strings on AdS(5) x S**5," Nucl. Phys. B 707, 303 (2005) arXiv:hep-th/0409086].

[10] D. Israel, C. Kounnas, D. Orlando and P. M. Petropoulos, "Electric / magnetic deformations of $\mathrm{S}^{* *} 3$ and $\operatorname{AdS}(3)$, and geometric Fortsch. Phys. 53, 73 (2005) arXiv:hep-th/0405213.

[11] O. Lunin and J. M. Maldacena, "Deforming field theories with U(1) x U(1) global symmetry and their gravity duals," JHEP 0505, 033 (2005) [arXiv:hep-th/0502086].

[12] W. Y. Wen, "Spin chain from marginally deformed AdS(3) x S**3," Phys. Rev. D 75, 067901 (2007) arXiv:hep-th/0610147.

[13] S. A. Frolov, R. Roiban and A. A. Tseytlin, "Gauge - string duality for superconformal defor- 
mations of N = 4 super Yang-Mills theory," JHEP 0507, 045 (2005) arXiv:hep-th/0503192.

[14] W. H. Huang, "Spin Chain with Magnetic Field and Spinning String in Magnetic Field Background," Phys. Rev. D 74, 027901 (2006) arXiv:hep-th/0605242.

[15] P. Bozhilov, "Probe branes dynamics: Exact solutions in general backgrounds," Nucl. Phys. B 656, 199 (2003) arXiv:hep-th/0211181.

[16] P. Bozhilov and R. C. Rashkov, "Magnon-like dispersion relation from M-theory," Nucl. Phys. B 768, 193 (2007) arXiv:hep-th/0607116.

[17] Q. Ding, "On the 1+2 dimensional Landau-Lifshitz equation," arXiv:math/0504288.

[18] N. Papanicolaou, "Duality Rotation For Two-Dimensional Classical Ferromagnets," Phys. Lett. Abf 84 (1981), 151-154.

[19] M. E. Gouvêa, G. M. Wysin, A. R. Bishop, F. G. Mertens, "Vortices in the classical twodimensional anisotropic Heisenberg model," Phys. Rev. B bf 39, 11840 (1989)

[20] F. G. Mertens and A. R. Bishop, "Dynamics of Vortices in Two-Dimensional Magnets," in P.L. Christiansen \& M.P. Sorensen, eds. Nonlinear Science at the Dawn of the 21st Century, Springer, 1999.

[21] T. Banks, W. Fischler, S. H. Shenker and L. Susskind, "M theory as a matrix model: A conjecture," Phys. Rev. D 55, 5112 (1997) arXiv:hep-th/9610043].

[22] T. Harmark, K. R. Kristjansson and M. Orselli, "Magnetic Heisenberg-chain / pp-wave correspondence," arXiv:hep-th/0611242.

[23] P. Bozhilov, "Neumann and Neumann-Rosochatius integrable systems from membranes on $A d S_{4} x S^{7}, "$ arXiv:0704.3082 [hep-th].

[24] J. Bjornsson and S. Hwang, "The membrane as a perturbation around string-like configurations," Nucl. Phys. B 689, 37 (2004) arXiv:hep-th/0403092. 